\title{
CRISPR/Cas9-mediated genome engineering of CXCR4 decreases the malignancy of hepatocellular carcinoma cells in vitro and in vivo
}

\author{
XIAOLI WANG, WENMEI ZHANG, YAN DING, XINGRONG GUO, YAHONG YUAN and DONGSHENG LI \\ Hubei Key Laboratory of Embryonic Stem Cell Research, Taihe Hospital, \\ Hubei University of Medicine, Shiyan, Hubei 442000, P.R. China
}

Received October 27, 2016; Accepted April 13, 2017

DOI: $10.3892 /$ or.2017.5601

\begin{abstract}
CXC chemokine receptor 4 (CXCR4) is associated with poor clinical outcomes and decreased survival in hepatocellular carcinoma (HCC). In the present study, we targeted CXCR4 by CRISPR/Cas9 in HepG2 cells and observed the effects both in vitro and in vivo. The results indicated that after targeting CXCR4 the expression of CXCR4 was significantly decreased and the cell proliferation was inhibited. Clonogenicity and scratch cell migration assays indicated that specific downregulation of CXCR4 inhibited cell migration. This disruption of CXCR4 led to less invasiveness, the genes related to epithelial-mesenchymal transition (EMT) and cell self-renewal were also affected. Moreover, sensitivity to the anticancer drug cisplatin was significantly increased in vitro by the downregulation of CXCR4. The results of the in vivo study showed that the growth volumes were significantly smaller in neoplasms derived from CXCR4-downregulated HepG2 cells compared to those derived from wild-type cells. These results showed that targeting CXCR4 by CRISPR/Cas9 could inhibit proliferation, migration and invasion, reversed EMT, increased chemosensitivity and decrease the malignancy of HCC in vitro and in vivo.
\end{abstract}

\section{Introduction}

$\mathrm{HCC}$ is the most common primary liver tumor and one of the most frequent malignant neoplasms worldwide (1). HCC is often not apparent, and thus many patients are diagnosed at advanced stages, which means that treatment is often less effective (2). These limited treatment options highlight the need to clarify the mechanisms of HCC development and

Correspondence to: Dr Dongsheng Li, Hubei Key Laboratory of Embryonic Stem Cell Research, Taihe Hospital, Hubei University of Medicine, Shiyan, Hubei 442000, P.R. China

E-mail: dsli698@126.com

Key words: CXC chemokine receptor 4, hepatocellular carcinoma, CRISPR/Cas9, malignancy, migration, invasion identify early disease biomarkers and new therapeutic targets. Chemokines play an essential role in tumor progression, and they have been reported to sustain tumor cell growth, induce angiogenesis and facilitate tumor escape through evasion of immune surveillance (3-6). Several studies have confirmed that the molecular pathogenesis of HCC involves chemokine/ chemokine receptors that participate in tumorigenesis and metastasis (7-9). CXCR4 is the most common chemokine receptor shown to be overexpressed in several cancers and to play an important role in tumorigenesis (10-12). Indeed, the level of CXCR4 expression serves as a prognostic measure of disease progression and survival. Although the receptor has been shown to be involved in HCC carcinogenesis, the specific role of CXCR4 in HCC remains incompletely understood. Thus, the molecular mechanism of CXCR4 in the progression of HCC warrants further investigation.

The recently developed genome-editing technique based on CRISPR/Cas9 is a broadly applicable approach for the efficient modification of essentially any sequence of interest in living cells or organisms (13). This system has the capability to quickly and efficiently disrupt genes, which often leads to translational termination and loss of gene function. In the present study, we targeted CXCR4 by CRISPR/Cas9 in the hepatoma cell line HepG2 to further elucidate the function of CXCR4 in HepG2 cells both in vitro and in vivo.

\section{Materails and methods}

Transfection and selection of stable luciferase-expressing HepG2 cells. The hepatoma cell line HepG2 was cultured in Dulbecco's modified Eagle's medium (DMEM; Gibco, Carsbad, CA, USA) supplemented with $10 \%$ fetal bovine serum (FBS) and incubated in a $37^{\circ} \mathrm{C}$ humidified atmosphere containing $5 \% \mathrm{CO}_{2}$. The pGL4.51(luc2/CMV/Neo) vector (Promega, Madison, WI, USA) was transfected into the cells using Lipofectamine 2000 (Invitrogen, Carlsbad, CA, USA) according to the manufacturer's instructions. At $24 \mathrm{~h}$ after transfection, the cell solution was diluted 1:10 and regenerated. A total of $400 \mathrm{mg} / \mathrm{ml} \mathrm{G} 418$ (Invitrogen) was added to the $10 \%$ DMEM culture medium for selection after the cells adhered to the plate. Limiting dilution was performed in a 96-well plate for repeated colony selection. 
CRISPR/Cas9-mediated downregulation of CXCR4. The gRNA-coding cDNAs for targeting CXCR4 gene were designed and synthesized to make the CXCR4-gRNA-Cas9 constructs. The primers including $20 \mathrm{bp}$ target sequence and $B s m$ BI sticky end were annealed and inserted into the pGK1.2 plasmid (Genloci Biotechnologies) digested with BsmBI (NEB). Primer sequences are as follows: sgRNA1, forward, 5'-CACCGCACTTCAGATAACTACACCG-3' and reverse 5'-AAACCGGTGTAGTTATCTGAAGTGC-3'; sgRNA2, forward, 5'-CACCGAAGCATGACGGACAAGTAC-3' and reverse, 5'-AAACGTACTTGTCCGTCATGCTTC-3'; sgRNA3, forward, 5'-CACCGGGCAATGGATTGGTCA TCC-3' and reverse, 5'-AAACGGATGACCAATCCATTGC CC-3'. Approximately $10 \mu$ l of CXCR4-gRNA-Cas9 plasmids $(10 \mu \mathrm{g})$ was mixed with $1 \times 10^{6} \mathrm{HepG} 2$ cells in a cuvette containing $90 \mu \mathrm{l}$ Opti-MEM. The transfection was performed under $150 \mathrm{~V}$ with a NEPA21 electroporator (Nepa Gene, Co., Ltd., Chiba, Japan). The transfected cells were transferred to a well of a 6 -well plate and cultured at $37^{\circ} \mathrm{C}$ with $3 \mu \mathrm{g} / \mathrm{ml}$ puromycin. After 3 days, the medium was replenished without the addition of puromycin, and the cells were maintained at $37^{\circ} \mathrm{C}$ for a few more days before they were harvested for DNA extraction and single-cell culture to select CXCR4-silenced cell clones.

T7E1 assay. Genomic DNA was prepared from wild-type HepG2 cells (control) and HepG2 CXCR4-downregulated cells (CXCR4) with the Genomic DNA Extraction Mini kit (Tiangen Biotech, Co., Ltd., Beijing, China). The genomic region that encompasses the target site was amplified by PCR, and its products were denatured and annealed to form heteroduplex DNA. The primer sequences to amplify the genomic region were as follows: forward, 5'-ACTATGGGAAAAGA TGGG-3' and reverse, 5'-ACTGCTGTAGAGGTTGACTG-3'; The annealed DNA was treated with 5 units of T7E1 (ViewSolid Biotechnology, Beijing, China) at $37^{\circ} \mathrm{C}$ for $15 \mathrm{~min}$; the DNA was then run on an agarose gel to separate the DNA fragments and identify the mutant clones. The target rate was calculated based on the intensities of DNA bands, which were proportionally measured by grey scale technique.

Cell proliferation assay. Cell proliferation assay was used to evaluate cell proliferation according to the manufacturer's instructions. The cells were seeded in 96-well plates at $1 \times 10^{4}$ cells/well and cultured for 24, 48, 72,96, 120 and $144 \mathrm{~h}$. A total of $20 \mu \mathrm{l}$ of CellTiter one solution reagent (Promega) was added to $100 \mu \mathrm{l}$ of medium in each well, the plates were incubated for $4 \mathrm{~h}$ at $37^{\circ} \mathrm{C}$, and the absorbance values were read by a $96-$-well plate reader at the absorbance of $490 \mathrm{~nm}$.

Western blot analysis. Cells from each group were collected, and proteins were extracted and separated by SDS-PAGE and then transferred onto PVDF membranes. After blocking with $1 \% \mathrm{BSA}$ for $1 \mathrm{~h}$ at room temperature, the membranes were incubated with antibodies specific for CXCR4 (1:200; Abcam, Cambridge, MA, USA), Bcl2 (1:200; Abcam), vimentin (1:200; Santa Cruz Biotechnology, Santa Cruz, CA, USA), E-cadherin (1:200; Santa Cruz Biotechnology), N-cadherin (1:200; Santa Cruz Biotechnology), MDR1 (1:200; Santa Cruz Biotechnology) and $\beta$-actin (1:1,000; Tiangen Biotech) over- night at $4^{\circ} \mathrm{C}$. After 3 washes in TBST, the membranes were incubated with alkaline phosphatase-conjugated secondary antibody (1:500; Tiangen Biotech) at room temperature for $1 \mathrm{~h}$. Then, the membranes were washed 3 times with TBST and imaged with a gel imaging system (Bio-Rad Laboratories, Hercules, CA, USA).

Clonogenicity assay. The clonogenicity of the cells was analyzed by colony-formation assay. The HepG2 cells with/without CXCR4 downregulation were plated onto a 12 -well plate pre-coated with $1 \%$ gelatin at a density of 1,000 cells/well. The cells were incubated at $37^{\circ} \mathrm{C}$ for 10 days and then the colonies were stained by crystal violet. The experiment was performed in triplicate.

Scratch cell migration assay. The scratch assay was used to observe cell migration. HepG2 cells with/without CXCR4 downregulation were cultured to complete confluence in 6-well plates, and a scratch was made across the cell monolayer in each well with a $10-\mu 1$ pipette tip. Then, the cell monolayer was washed 3 times with phosphate-buffered saline (PBS) and incubated in serum-free DMEM at $37^{\circ} \mathrm{C}$ with $5 \% \mathrm{CO}_{2}$ for 24 or $48 \mathrm{~h}$. The width of the scratches was measured and the percentages by which the width decreased were compared at 0,24 and $48 \mathrm{~h}$. The experiment was performed in triplicate.

Invasion assay. Cell invasion assay was performed using the Transwell chamber (8- $\mu \mathrm{m}$; Corning Life Sciences, Corning, NY, USA). The interior of the inserts precoated with $10 \mathrm{mg} / \mathrm{ml}$ growth factor-reduced Matrigel (BD Biosciences, Franklin Lakes, NJ, USA). Cells $\left(2 \times 10^{4}\right)$ were added to the interior of the inserts in $0.2 \mathrm{ml}$ of serum-free growth medium. Growth medium $(0.8 \mathrm{ml})$ supplemented with $10 \%$ bovine calf serum (BCS) was added to the lower chamber. After incubation for $48 \mathrm{~h}$ at $37^{\circ} \mathrm{C}$, the cells attached to the upper surface of the filter were removed with a cotton swab, and migrated cells on the lower surface of the filter were fixed and stained for 15 min with $0.25 \%$ crystal violet (Sigma-Aldrich, St. Louis, MO, USA), $10 \%$ formaldehyde and $80 \%$ methanol, and then the inserts were washed five times with $\mathrm{ddH}_{2} \mathrm{O}$ and photographed. Migrated cells were determined by counting cells in five microscopic fields per well, and the extent of migration was expressed as an average number of cells per microscopic field. Cells were imaged by phase contrast microscopy.

Quantitative PCR. Total RNA was extracted using TRIzol reagent (Ambion, Austin TX, USA) according to the manufacturer's recommendations and was quantified by UV spectroscopy. To prepare the RNA for PCR analysis, $2 \mu \mathrm{g}$ total RNA was converted to cDNA using the FastQuant RT kit with gDNase (Tiangen Biotech). Quantitative PCR was then conducted with a SYBR-Green Master Mix (Takara Bio, Dalian, China). The PCR reaction proceeded as follows: $95^{\circ} \mathrm{C}$ for $30 \mathrm{sec}$, followed by 40 cycles of $95^{\circ} \mathrm{C}$ for $30 \mathrm{sec}, 60^{\circ} \mathrm{C}$ for $30 \mathrm{sec}$ and $72^{\circ} \mathrm{C}$ for $30 \mathrm{sec}$. The gene expressions were normalized to GAPDH. The primer sequences for the genes were as follows: GAPDH, forward, 5'-CCAAGGTCATCCATGACA AC-3' and reverse, 5'-TGTCATACCAGGAAATGAGC-3'; Snail, forward, 5'-GAAAGGCCTTCAACTGCAAA-3' and reverse, 5'-TGACATCTGAGTGGGTCTGG-3'; Slug, forward, 
A

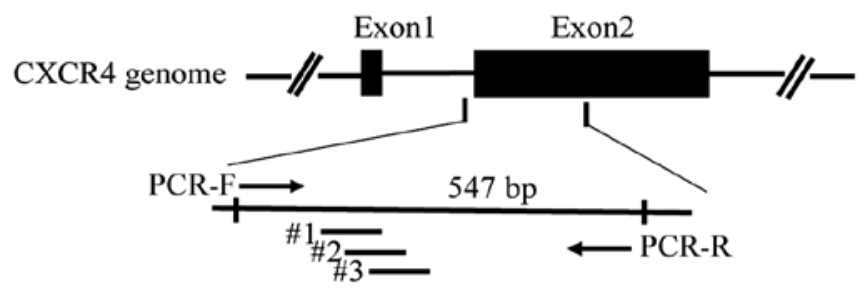

B

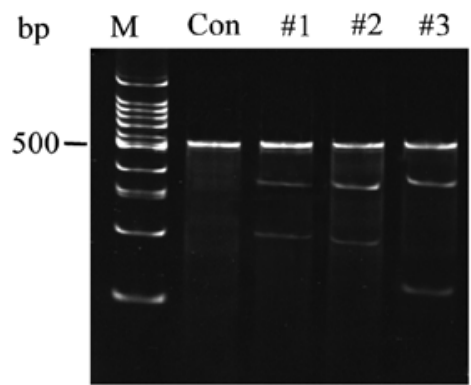

C \#3

PAM

TACCAGAAGAAACTGAGAAGCATGACGGACAAGTACAGGCTGCACCTGTC TACCAGAAGAAACTGAGAAGCATGACGG---------TACAGGCTGCACCTGTC wild type

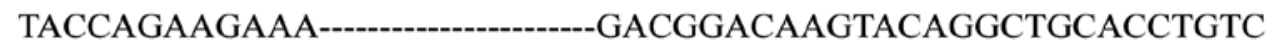
$-5 \mathrm{bp}$ TACCAGAAGAAACTGAGAAGCATGACGGA---------ACCTGTC $-11 \mathrm{bp}$ $-14 \mathrm{bp}$ TACCAGAAGAAACTGAGAAGCATGACGGACAA----ACAGGCTGCACCTGTC $-2 \mathrm{bp}$ TACCAGAAGAAACTGAGAAGCAT--------------AAGTACAGGCTGCACCTGTC TACCAGAAGAAACTGAGAAGCATGACGGACAAG-----CAGGCTGCACCTGTC TACCAGAAGAAACTGAGAAACGGACAAGTACAGGCTGCACCTGTC

$-7 \mathrm{bp}$

$-2 \mathrm{bp}$ $-5 \mathrm{bp}$

D TACCAGAAGAAACTGAGAAGCATGACGGACAAGTACAGGCTGCACCTGTC $0 \mathrm{bp}$ TACCAGAAGAAACTGAGAAGCATGACG----------GTACAGGCTGCACCTGTC $-5 \mathrm{bp}$ TACCAGAAGAAACTGAGAAGCATGACGGACAAGTAC-----GCTGCACCTGTC $-2 \mathrm{bp}$ TACCAGAAGAAACTGAGGACAAGTACAGGCTGCACCTGTC

$-10 \mathrm{bp}$

Figure 1. CRISPR/Cas9-mediated CXCR4 downregulation in HepG2 cells. (A) Schematic diagram of gRNAs targeting the CXCR4 locus. (B) T7E1 assay of each gRNA-CRISPR/Cas9 targeting CXCR4 in HepG2 cells. The genomes were amplified by PCR and then digested with T7E1. M, 100 bp DNA ladder. Control, HepG2 cells. Lanes \#1, \#2 and \#3, HepG2 cells targeted by different gRNAs-CRISPR/Cas9. (C) Gene sequence of the mutated CXCR4 gene at the target sites of \#3 gRNA-CRISPR/Cas9. The number of deleted nucleotides (dashes) is marked on the right end of each sequence. (D) Sequencing of the CXCR4 mutant single-cell clone after targeting by CRISPR/Cas9.

\section{5'-AGATGCATATTCGGACCCAC-3' and reverse, 5'-CCTC ATGTTTGTGCAGGAGA-3'.}

Chemosensitivity assay. HepG2 cells with/without CXCR4 downregulation were seeded onto a 96 -well plate $\left(1 \times 10^{4}\right.$ cells/well) and incubated at $37^{\circ} \mathrm{C}$ for $24 \mathrm{~h}$. The cells received fresh medium that contained $1 \mu \mathrm{g} / \mathrm{ml}$ cisplatin, and the cells were continuously incubated for an additional $48 \mathrm{~h}$; the cells were then removed from the incubator and treated with $1 \mu \mathrm{l}$ luciferase substrate, which was added to the medium for 10-15 min. The viability of the cells was examined using a fluorescence imaging system (IVIS ${ }^{\circledast}$ Spectrum; Caliper Life Sciences, Inc., Waltham, MA, USA). The experiment was performed in triplicate.

Xenograft assay. HepG2 cells with/without CXCR4 downregulation at a density of $5 \times 10^{6}$ were subcutaneously injected into 4 - to 6 -week-old nude mice (3 mice per group). These mice were bred, and the sizes of the neoplasms from the implanted cells were examined weekly using the IVIS Spectrum system (Caliper Life Sciences, Waltham, MA, USA). Mice were anesthetized by inhalation of $2 \%$ isoflurane and then received an intra-peritoneal injection of D-luciferin; bioluminescence images were obtained $15 \mathrm{~min}$ after the luciferin injection. The bioluminescence signal was analyzed with software after the placement of a small region of interest. The mean light intensity was then measured within this region of interest. The research protocol on animals was approved by the Ethics Committee of the First Affiliated Taihe Hospital of Hubei University of Medicine.

Statistical analysis. The results are presented as the mean \pm standard deviation (SD). The statistical significance of the differences was tested with the Student's t-test in Microsoft Office Excel. In the comparisons, values of $\mathrm{P}<0.05$ were considered statistically significant.

\section{Results}

T7E1 analysis and selection of CXCR4 mutant cell clones. In order to genetically disrupt the CXCR4 allele, we designed 3 gRNAs to target Cas9 to the conserved sites of human CXCR4 gene (Fig. 1A) and generated CXCR4-gRNA-Cas9 plasmids to deliver gRNAs into HepG2 cells. The target region of the CXCR4 gene was amplified by PCR and then digested with the T7E1 enzyme. We found that the three gRNAs induced CXCR4 mutations at a rate of 23.5, 25 and $29.5 \%$, respectively (Fig. 1B). We chose \#3 gRNA for the following experiment, different mutations were found according to the gene sequencing of 30 single-cell clones (Fig. 1C); in particular, one cell clone with triallelic CXCR4 mutations (CXCR4) was selected by gene sequencing (Fig. 1D). At least three CXCR4 alleles were mutated in this HepG2 cell clone, and this clone was therefore used for subsequent experiments. 

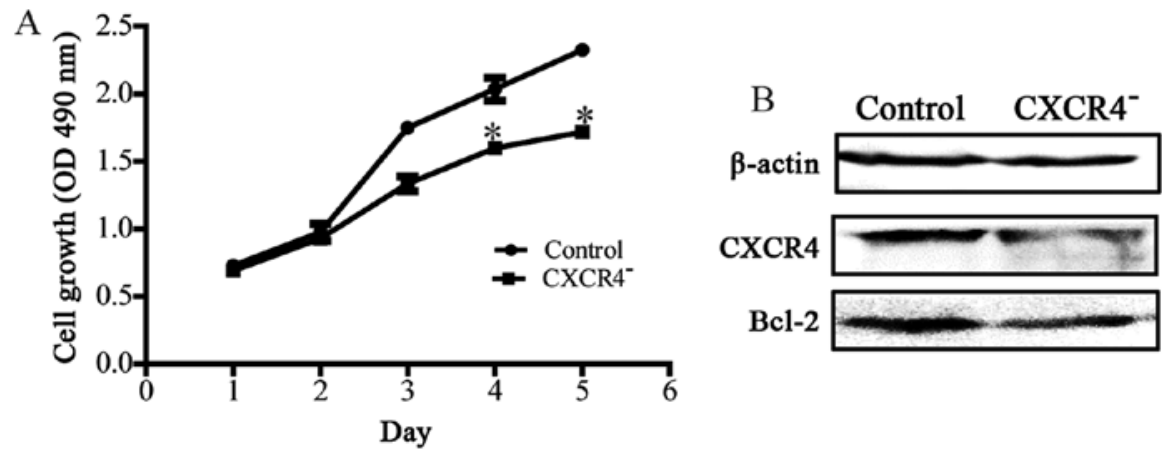

Figure 2. Cell proliferation of HepG2 and CXCR4- HepG2 cells. (A) Detection of the proliferation by MTT ("P<0.05). (B) Analysis of proliferation and apoptosis-associated protein by western blotting.

A

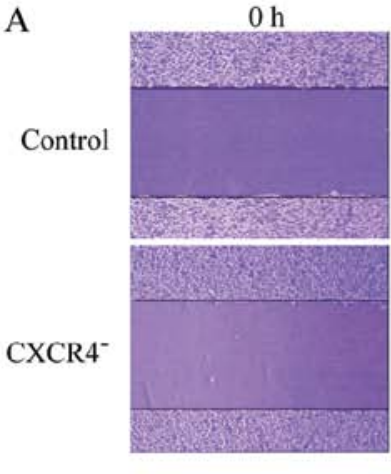

$\mathrm{C}$

Control
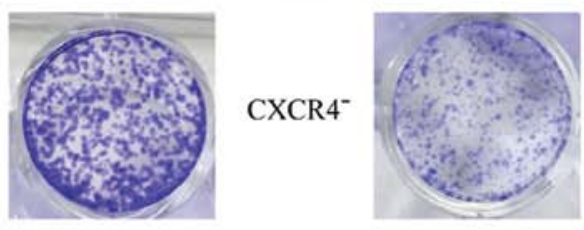

B

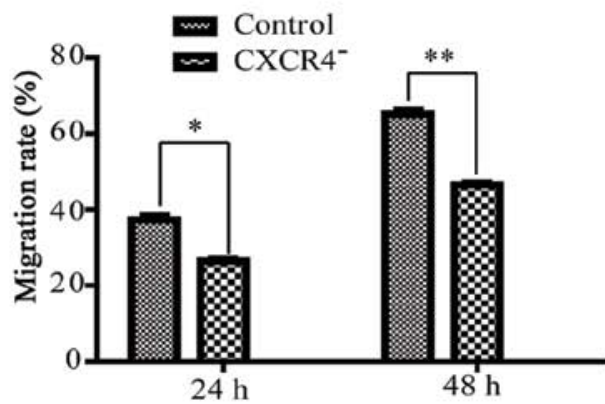

D

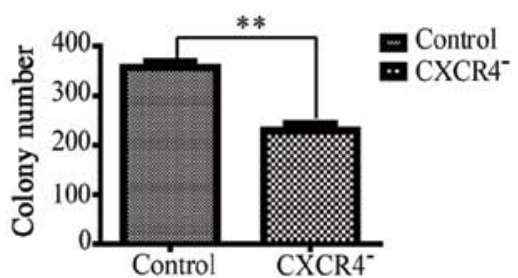

Figure 3. Scratch assay and clonogenicity of HepG2 and CXCR4- HepG2 cells. (A) Images were obtained at 0,24 and 48 h for wild-type HepG2 cells and CXCR4- HepG2 cells after the scratch was made. (B) Comparison of the migration distances between HepG2 cells and CXCR4- HepG2 cells at 24 and $48 \mathrm{~h}$. (C) Image of the cell clones formed from dissociated HepG2 cells and CXCR4- HepG2 cells after 10 days of incubation at a density of 1,000 cells/well in a 12-well plate. (D) Comparison of the cell colony numbers formed from dissociated HepG2 cells and CXCR4- HepG2 cells after 10 days of incubation. Data are presented as the mean \pm SEM of three independent experiments $\left(\right.$ ( $\mathrm{P}<0.05$ and $\left.{ }^{* *} \mathrm{P}<0.001\right)$.

Downregulation of CXCR4 inhibits the proliferation of HepG2 cells. The impact of downregulating CXCR4 expression on cell proliferation was detected by MTT. The number of proliferating cells was determined by measuring the optical density (OD) value at $490 \mathrm{~nm}$. The detection results indicated that the speed of cell proliferation was slower in the CXCR4 group than the control group after cultured for 4 days $(\mathrm{P}<0.05$; Fig. 2A). We also detected the proliferation and apoptosis associated protein $\mathrm{Bcl} 2$ by western blot analysis, the result showed that downregulation of CXCR4 could decrease the expression level of $\mathrm{Bcl} 2$ protein (Fig. 2B).

Downregulation of CXCR4 restricts the migration and cloning efficiency of HepG2 cells. According to the scratch assay, the migration rates of CXCR4- $4^{-}$epG2 cells at 24 and $48 \mathrm{~h}$ were $24 \pm 3$ and $45 \pm 4 \%$, respectively, while those for wild-type HepG2 cells were $36 \pm 4$ and $64 \pm 7 \%$, respectively (Fig. 3A and B). This significant difference $(\mathrm{P}<0.05)$ indicated that the migration of HepG2 cells was strongly inhibited by CXCR4 downregulation. In the cell colony formation assay, as shown in Fig. 3C and D, the number of colonies formed by CXCR4- HepG 2 cells was $229 \pm 10$, while that formed by wild-type HepG2 cells was $356 \pm 14$. This significant difference $(\mathrm{P}<0.05)$ indicated that the clonogenicity of $\mathrm{HepG} 2$ cells was strongly inhibited by CXCR4 downregulation.

Downregulation of CXCR4 alleviates the invasiveness of the HepG2 cells. Transwell cell migration assay was carried out to investigate how the invasiveness of HepG2 cells was affected by CXCR4 disruption. As shown in Fig. 4, the numbers of the cells that passed through the Matrigel at $48 \mathrm{~h}$ was $12 \pm 3$ for CXCR4-HepG2 cells and $78 \pm 7$ for the HepG2 cells. The number for the downregulation of CXCR4 in HepG2 cells was remarkably less than the wild-type $(\mathrm{P}<0.05)$.

Downregulation of CXCR4 in HepG2 cells reverses the status of EMT. EMT is a critical event that can be observed during tumor progression. Quantitative RT-PCR analysis revealed that the expression levels of some EMT-regulated transcription factor genes, such as Slug and Snail, were downregulated 

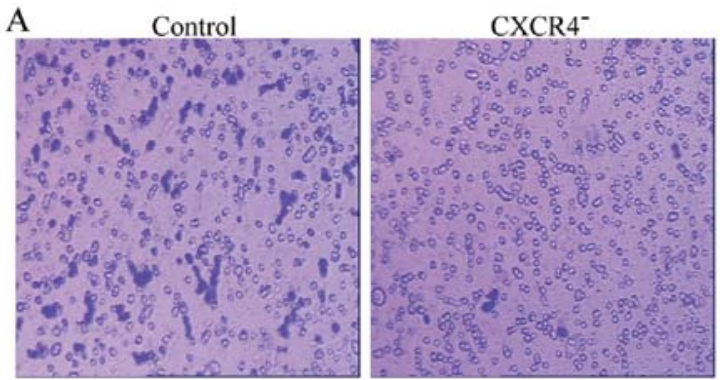

B

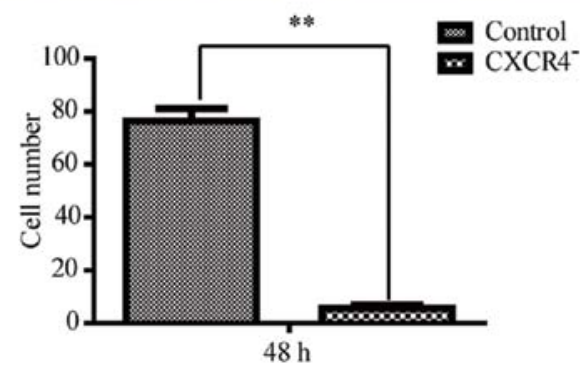

Figure 4. Transwell assay of HepG2 and CXCR4- HepG2 cells. (A) Images were obtained at $48 \mathrm{~h}$ for HepG2 cells and CXCR4- HepG2 cells. (B) Comparison of the cell numbers that passed the membrane of Transwell at $48 \mathrm{~h}$. Data are presented as the mean \pm SEM of three independent experiments $\left({ }^{*} \mathrm{P}<0.05\right.$ and $\left.{ }^{* *} \mathrm{P}<0.001\right)$.

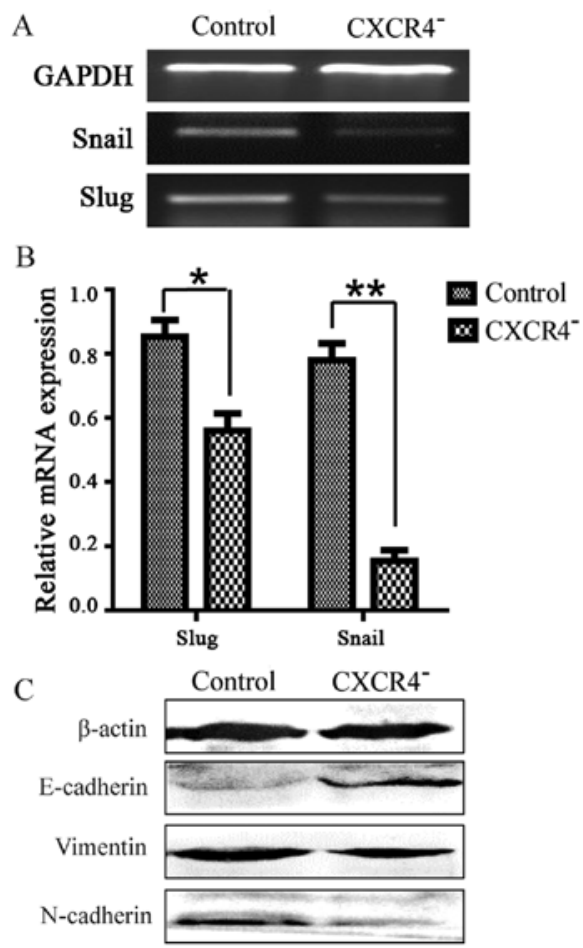

Figure 5. Reversal of EMT in CXCR4- HepG2 cells. (A) RT-PCR results for EMT-related transcription factor genes. (B) Real-time PCR results for several EMT-related transcription factor genes. (C) Detection of the expression of the epithelial marker E-cadherin and the mesenchymal markers $\mathrm{N}$-cadherin and vimentin by western blotting.

in CXCR4- HepG2 cells (Fig. 5A and B). At the protein level, upregulation of the epithelial marker E-cadherin and downregulation of the mesenchymal markers vimentin and $\mathrm{N}$-cadherin were also observed (Fig. 5C). Together, these changes suggested a reversal of EMT.
A

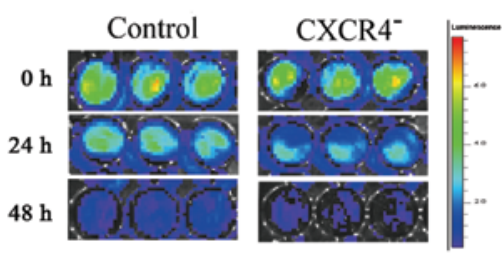

B

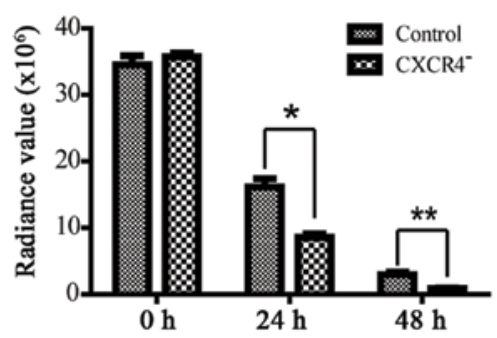

C

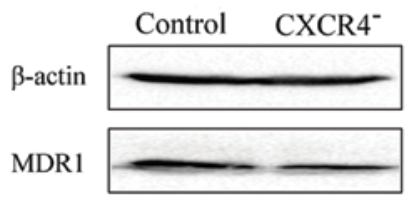

Figure 6. Chemosensitivity assay. (A) Fluorescence imaging of cell viability in HepG2 and CXCR4 ${ }^{-}$HepG2 cells after exposure to cisplatin $(1 \mu \mathrm{g} / \mathrm{ml})$ for 24 or $48 \mathrm{~h}$. (B) Comparisons of the cell viabilities after exposure to cisplatin. (C) Detection of the expression level of MDR1 protein by western blotting. Control signifies wild-type HepG2 cells; CXCR4 signifies cells where the expression of CXCR4 was disrupted. Data are presented as the mean \pm SEM of three independent experiments ( $\mathrm{P}<0.05$ and $\left.{ }^{* *} \mathrm{P}<0.001\right)$.

Downregulation of CXCR4 increases the sensitivity of HepG2 cells to cisplatin in vitro. The chemosensitivity of the HepG2 and the CXCR4- HepG2 cells was compared according to their viability after exposure to cisplatin. As shown in Fig. 6A and B, CXCR4- HepG2 cells were more sensitive to cisplatin. MDR1, which is an important indicator of drug resistance in chemotherapy, was also significantly downregulated in CXCR4- HepG2 cells (Fig. 6C).

Downregulation of CXCR4 decreases the growth of HepG2 xenograft tumor in nude mice. To further investigate the inhibitory effect of CXCR4- HepG2 cells on tumor cell growth in vivo, we used a xenograft cancer model. Either CXCR4- HepG2 cells or wild-type HepG2 cells were injected subcutaneously into nude mice, and the sizes of the neoplasms that formed from the implanted HepG2 cells were measured weekly (Fig. 7A). Based on our results, the sizes of the neoplasms formed from the CXCR4- HepG2 cells were significantly smaller than those formed from wild-type cells (Fig. 7B).

\section{Discussion}

The development of a malignant tumor is a complex process that involves multiple factors and stages as well as malfunctions or mutations in a variety of genes. CRISPR/Cas9 is a useful tool that can be used to study the function of genes (14). Although CXCR4 has been shown to be involved in many malignant human tumors, few studies have investigated the effects of the directed inhibition of CXCR4 in HCC. To gain additional insight into the effects of CXCR4 in HepG2 
A

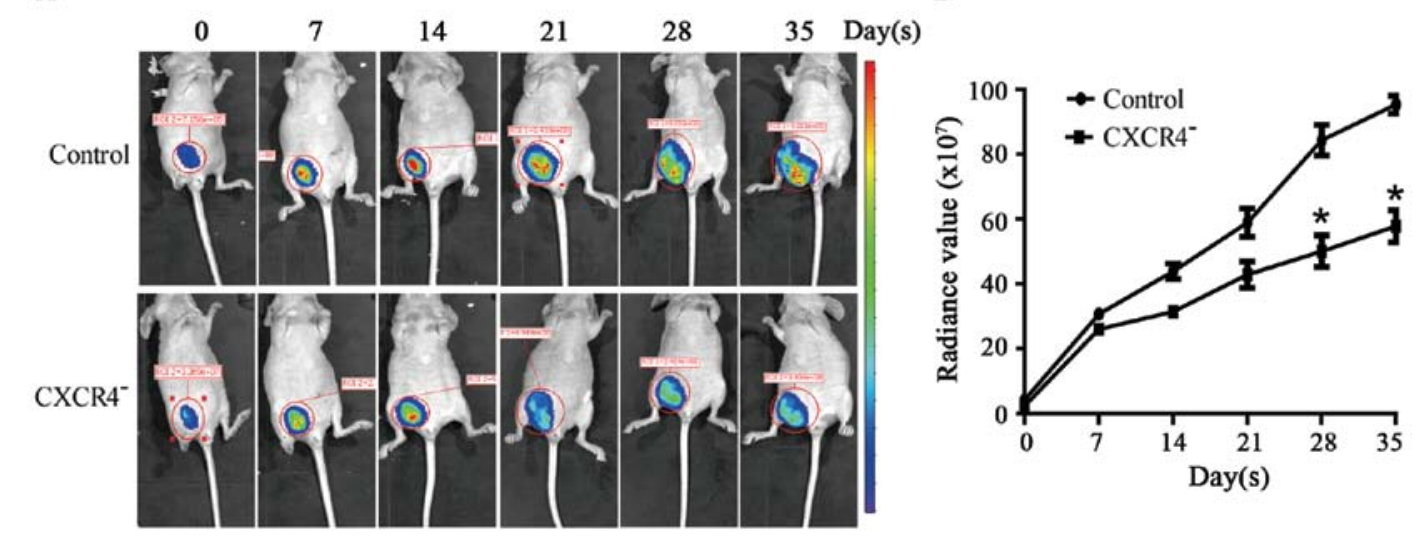

B

Figure 7. Xenograft test. HepG2 cells and CXCR4- ${ }^{-}$epG2 cells $\left(5 \times 10^{6}\right.$ each) were subcutaneously injected into the flanks of nude mice ( $\left.\mathrm{n}=3\right)$, and the sizes of the neoplasms that formed were measured with an in vivo imaging system (for a period of 6-8 weeks). (A) Serial images obtained at different time-points. (B) The changes in the values of the fluorescein signal as the neoplasms formed. Data are presented as the mean \pm SEM of three independent experiments $($ ( $\mathrm{P}<0.05)$.

cells, we selectively knocked down CXCR4 expression using CRISPR/Cas9 and observed the effects both in vitro and in vivo. In the present study, CXCR4 was effectively disrupted by CRISPR/Cas9, and the expression of CXCR4 was remarkably downregulated and the cell proliferation was inhibited.

The grade of malignancy is closely related to the migration clonogenicity and invasion of tumor cells. In the present study, scratch assays showed that the migration and invasiveness of CXCR4- HepG2 cells were significantly restricted, and colony formation assays showed that the clonogenicity of HepG2 cells with knocked down CXCR4 expression was decreased. These findings suggested that CXCR4- HepG2 cells were less malignant than their wild-type counterparts.

EMT may indicate a progression toward malignancy, with increased potential for invasion and metastasis (15-17). During this process, cancer cells acquire a fibroblast-like phenotype (18). However, in the present study, this progression seemed to be markedly reversed by the downregulation of CXCR4. After downregulation of CXCR4 in HepG2 cells, the expression levels of several genes that are critical for progression from an epithelial to a mesenchymal phenotype were altered. Specifically, we observed the downregulation of mesenchymal markers such as vimentin and snail and the upregulation of epithelial markers such as E-cadherin. These data suggested that CXCR4 may play a critical role in the process of EMT. An understanding of the molecular mechanisms responsible for EMT and the discovery of approaches to reverse this process should be very important for the development of new therapeutic strategies against HCC.

The cytotoxicity of cisplatin to normal tissues and the acquired resistance of cancer cells to this drug have reduced its clinical efficacy (19). However, the exact mechanism of cisplatin resistance is not clear. MDR1 is the most important indicator for chemoresistance $(20,21)$. Our experiments result revealed that tumor $\mathrm{CXCR} 4$ can modulate cisplatin sensitivity by influence the expression level of MDR1 and the downregulation of CXCR4 in HepG2 cells increased their sensitivity to cisplatin in vitro.
In conclusion, we used CRISPR/Cas9 to efficiently dowregulate CXCR4 expression, which led to a marked decrease in the grade of malignancy of HCC in vitro and in vivo. CRISPR/Cas9-mediated genome engineering may be a good way to study the function of the potential oncogenes on the genome level and provide a rapid avenue for functional cancer genomics study in the future.

\section{Acknowledgements}

This study was supported by the National Natural Science Foundation of Hubei province (2012FFA037), the Natural Science Foundation of Hubei Provincial Department of Education (Q20162110), the Key Science and Technology Project of Hubei Province (2016ACA157) and the National Natural Science Foundation of China (81641028).

\section{References}

1. Flores A and Marrero JA: Emerging trends in hepatocellular carcinoma: focus on diagnosis and therapeutics. Clin Med Insights Oncol 8: 71-76, 2014.

2. Shinoda M, Kishida N, Itano O, Ei S, Ueno A, Kitago M, Abe Y, Hibi T, Yagi H, Masugi Y, et al: Long-term complete response of advanced hepatocellular carcinoma treated with multidisciplinary therapy including reduced dose of sorafenib: Case report and review of the literature. World J Surg Oncol 13: 144, 2015.

3. Gassmann P, Haier J, Schlüter K, Domikowsky B, Wendel C, Wiesner U, Kubitza R, Engers R, Schneider SW, Homey B, et al: CXCR4 regulates the early extravasation of metastatic tumor cells in vivo. Neoplasia 11: 651-661, 2009.

4. Hattermann K, Holzenburg E, Hans F, Lucius R, Held-Feindt J and Mentlein R: Effects of the chemokine CXCL12 and combined internalization of its receptors CXCR4 and CXCR7 in human MCF-7 breast cancer cells. Cell Tissue Res 357: 253-266, 2014.

5. Zhou SL, Dai Z, Zhou ZJ, Wang XY, Yang GH, Wang Z, Huang XW, Fan J and Zhou J: Overexpression of CXCL5 mediates neutrophil infiltration and indicates poor prognosis for hepatocellular carcinoma. Hepatology 56: 2242-2254, 2012.

6. Wendt MK, Johanesen PA, Kang-Decker N, Binion DG, Shah V and Dwinell MB: Silencing of epithelial CXCL12 expression by DNA hypermethylation promotes colonic carcinoma metastasis. Oncogene 25: 4986-4997, 2006. 
7. Schimanski CC, Bahre R, Gockel I, Müller A, Frerichs K, Hörner V, Teufel A, Simiantonaki N, Biesterfeld S, Wehler T, et al: Dissemination of hepatocellular carcinoma is mediated via chemokine receptor CXCR4. Br J Cancer 95: 210-217, 2006.

8. Chen RX, Song HY, Dong YY, Hu C, Zheng QD, Xue TC, Liu XH, Zhang Y, Chen J, Ren ZG, et al: Dynamic expression patterns of differential proteins during early invasion of hepatocellular carcinoma. PLoS One 9: e88543, 2014.

9. Xiang ZL, Zeng ZC, Tang ZY, Fan J, Zhuang PY, Liang Y, Tan YS and He J: Chemokine receptor CXCR4 expression in hepatocellular carcinoma patients increases the risk of bone metastases and poor survival. BMC Cancer 9: 176, 2009.

10. Ehtesham M, Winston JA, Kabos P and Thompson RC: CXCR4 expression mediates glioma cell invasiveness. Oncogene 25 : 2801-2806, 2006.

11. Andre F, Xia W, Conforti R, Wei Y, Boulet T, Tomasic G, Spielmann M, Zoubir M, Berrada N, Arriagada R, et al: CXCR4 expression in early breast cancer and risk of distant recurrence. Oncologist 14: 1182-1188, 2009.

12. Blot E, Laberge-Le Couteulx S, Jamali H, Cornic M, Guillemet C, Duval C, Hellot MF, Pille JY, Picquenot JM and Veyret C: CXCR4 membrane expression in node-negative breast cancer. Breast J 14: 268-274, 2008.

13. Hsu PD, Lander ES and Zhang F: Development and applications of CRISPR-Cas9 for genome engineering. Cell 157: 1262-1278, 2014.

14. Gori JL, Hsu PD, Maeder ML, Shen S, Welstead GG and Bumcrot D: Delivery and specificity of CRISPR-Cas9 genome editing technologies for human gene therapy. Hum Gene Ther 26 : 443-451, 2015.
15. Katoh M: Epithelial-mesenchymal transition in gastric cancer (Review). Int J Oncol 27: 1677-1683, 2005.

16. Rhim AD, Mirek ET, Aiello NM, Maitra A, Bailey JM, McAllister F, Reichert M, Beatty GL, Rustgi AK, Vonderheide RH, et al: EMT and dissemination precede pancreatic tumor formation. Cell 148: 349-361, 2012.

17. Creighton CJ, Chang JC and Rosen JM: Epithelial-mesenchymal transition (EMT) in tumor-initiating cells and its clinical implications in breast cancer. J Mammary Gland Biol Neoplasia 15: 253-260, 2010.

18. Huber MA, Kraut N and Beug H: Molecular requirements for epithelial-mesenchymal transition during tumor progression. Curr Opin Cell Biol 17: 548-558, 2005.

19. Dasari S and Tchounwou PB: Cisplatin in cancer therapy: molecular mechanisms of action. Eur J Pharmacol 740: 364-378, 2014.

20. Nakayama K, Kanzaki A, Ogawa K, Miyazaki K, Neamati N and Takebayashi Y: Copper-transporting P-type adenosine triphosphatase (ATP7B) as a cisplatin based chemoresistance marker in ovarian carcinoma: Comparative analysis with expression of MDR1, MRP1, MRP2, LRP and BCRP. Int J Cancer 101: 488-495, 2002.

21. Su F, Ouyang N, Zhu P, Ouyang N, Jia W, Gong C, Ma X, Xu H and Song E: Psychological stress induces chemoresistance in breast cancer by upregulating mdrl. Biochem Biophys Res Commun 329: 888-897, 2005. 